\title{
Optimización de Hidrólisis Enzimática de la Fracción Globular de Sangre Bovina por Metodología de Superficie Respuesta y Evaluación de sus Propiedades Antioxidantes
}

\author{
Jesús A. Morales ${ }^{(1)^{*}}$, Omar A. Figueroa ${ }^{(2)}$ y José E. Zapata(3) \\ (1) Universidad Popular del Cesar, Facultad de Ingenierías y Tecnologías. Grupo GEAB. Balneario \\ Hurtado Vía a Patillal- oficina 205; Valledupar, Cesar - Colombia. \\ (e-mail: jesusmoralesg@unicesar.edu.co) \\ (2) Universidad de la Guajira, Programa de Ingeniería Industrial. Kilómetro 5 vía Maicao, Riohacha - \\ Colombia. (e-mail: ofigueroam@uniguajira.edu.co) \\ (3) Universidad de Antioquia, Grupo de Nutrición y tecnología de Alimentos. Calle 67 No. 53-108, \\ Medellín - Colombia. (e-mail: edgar.zapata@udea.edu.co)
}

Recibido Jul. 6, 2016; Aceptado Sep. 9, 2016; Versión final Nov. 3, 2016, Publicado Abr. 2017

\begin{abstract}
Resumen
En este trabajo se optimizó la reacción de hidrólisis enzimática de proteínas de la fracción globular de sangre bovina usando la metodología de superficie de respuesta. Se evalúo el efecto de la concentración de sustrato $\left(\mathrm{S}_{0}: 8-12 \%\right)$, la relación enzima/sustrato $\left(\mathrm{E}_{0} / \mathrm{S}_{0}: 4-12 \mathrm{~g} / 100 \mathrm{~g}\right.$ proteína) y el pH (8-10), sobre el grado de hidrólisis $(\mathrm{GH})$, en un reactor Batch (temperatura de $55^{\circ} \mathrm{C}$, por $2 \mathrm{~h}$ ). La optimización del modelo estadístico obtenido entregó un máximo GH de 38,08 \% para pH: 8,16; $\mathrm{S}_{0}: 6,6$ y $\mathrm{E}_{0} / \mathrm{S}_{0}: 14,7$, el cual fue corroborado experimentalmente dando un GH de 36,25 $\pm 0,3$ con sesgo negativo de $5 \%$. A estas condiciones se tomaron muestras a tres tiempos de hidrólisis para evaluar la capacidad antioxidante, con los métodos químicos conocidos como ORAC, ABTS y FRAP. Se alcanzó mayor capacidad antioxidante a mayor tiempo de hidrólisis. Después de una digestión in vitro se corroboró que la capacidad antioxidante del hidrolizado se conserva.
\end{abstract}

Palabras clave: péptidos bioactivos; hidrólisis enzimática; actividad antioxidante; sangre bovina; proteína

\section{Optimization of Enzymatic Hydrolysis of Bovine Blood Cell Fraction by Response Surface Methodology (RSM) and Evaluation of its Antioxidant Properties}

\begin{abstract}
In this paper the reaction of enzymatic hydrolysis of the erythrocyte fraction of bovine blood was optimized through the response surface methodology. The effects of substrate concentration ( $\left.\mathrm{S}_{0}: 8-12 \%\right)$, enzyme/substrate ratio $\left(\mathrm{E}_{0} / \mathrm{S}_{0}: 4-12 \mathrm{~g} / 100 \mathrm{~g}\right.$ protein) and $\mathrm{pH}(8-10)$, on degree of hydrolysis $(\mathrm{DH})$ working to constant temperature of $55^{\circ} \mathrm{C}$ in a batch reactor, by $2 \mathrm{~h}$ were determined. The optimization of the statistical model obtained gave a maximum $\mathrm{DH}$ of $38.08 \%$, with $\mathrm{pH} 8.16 ; \mathrm{S}_{0}: 6.6$ and $\mathrm{E}_{0} / \mathrm{S}_{0}: 14.7$, which was experimentally proved giving a DH of $36.25 \pm 0.3$ with negative bias of $5 \%$. At the optimal conditions samples were taken at three hydrolysis times to evaluate the antioxidant capacity, which was analyzed with ORAC, ABTS and FRAP methods. Greater antioxidant capacity was obtained at long hydrolysis time. After in vitro digestion it was confirmed that the antioxidant capacity of hydrolyzate remained.
\end{abstract}

Keywords: bioactive peptides; enzymatic hydrolysis; antioxidant activity; bovine blood; protein 


\section{INTRODUCCIÓN}

Desde el momento que se empieza la acumulación de materia prima de origen biológico para el acondicionamiento y la transformación en productos de consumo por parte de la agroindustria, hasta las últimas etapas de los diferentes procesos, se generan diversos subproductos que al no ser aprovechados se convierten en un problema para las empresas procesadoras (Laca et al., 2004). En el caso de la industria cárnica se generan subproductos como sangre, tendones, astillas de huesos y diferentes recortes, que realmente no tienen una demanda clara en el mercado. La sangre proveniente de sacrificio animal representa una parte significativa de estos subproductos, alrededor de 4 \% de peso vivo del animal (Laca et al., 2004; Bah et al., 2013).

La sangre es una fuente rica en proteínas de alta calidad, pero muy poco valorada en el mercado, por tal razón diferentes investigaciones han estudiado los hidrolizados de sangre de diferentes animales de consumo, con el fin de evaluar la capacidad biológica de los péptidos resultantes de sus fracciones (Bah et al., 2013). En la última década se ha establecido que algunos péptidos obtenidos por hidrólisis de proteínas alimentarias, son capaces de ejercer efectos biológicos específicos, tales como: antimicrobiano, antiviral, anticancerígeno, opioide, antioxidante, antihipertensivo y antitrombótico (Gomes et al., 2010; Liu et al., 2010; Möller et al., 2008), mientras que en el caso de la sangre se ha podido evaluar principalmente su capacidad antioxidante y antihipertensiva (Bah et al., 2013). Estas actividades dependen principalmente de factores como el tipo de proteasa utilizada, las condiciones de la hidrólisis, la composición en aminos- ácidos, la secuencia y configuración de los péptidos (Tavano, 2013). Obtener hidrolizados con altos grados de hidrólisis (GH), es de interés cuando de buscar péptidos bioactivos se trata, debido a la relación entre la actividad biológica de los péptidos y su peso molecular (Saidi et al., 2013; Wang et al., 2013; Liu et al., 2014; Gómez et al., 2013), siendo las fracciones con pesos moleculares entre 1 - 4kDa los más interesantes para usos nutricionales y/o farmacéuticos (Saidi et al., 2014; de Castro y Sato, 2015; Opheim et al., 2015).

Buscando comprender el proceso de hidrólisis enzimática de proteínas se han planteado diferentes modelos para representar la cinética enzimática de proteínas de fuentes alimentarias, como caseínas, lactoalbumina, hemoglobina bovina y sustratos de origen vegetal, generando información básica para la optimización de procesos (Figueroa et al., 2012). Además se han estudiado los modelos y mecanismos enzimáticos considerando la inhibición de enzimas y relacionándolo con las constantes cinéticas, obteniendo buenos ajustes para las curvas de cinéticas (Márquez-Moreno y Fernández-Cuadrado, 1993; Qi y He, 2006; Valencia et al., 2014; Valencia et al., 2015). Mientras que otros estudios han demostrado que el pH y la concentración de sustratos son claves en análisis de la hidrólisis enzimática de proteínas de sangre porcina y bovina (Chang, Wu, et al. 2007; Figueroa, O et al. 2016).

Los péptidos bioactivos de diferentes fuentes, han encontrado aplicación en la industria nutraceutica y farmacéutica, principalmente para la elaboración de alimentos funcionales. En esta investigación se buscó la optimización del proceso de hidrólisis de la fracción globular de la sangre bovina (FG) con respecto al grado de hidrólisis $\mathrm{GH}$, analizando la cinética enzimática con relación a las variables más importantes del proceso $\left(\mathrm{pH}, \mathrm{S}_{0}\right.$ y $\left.\mathrm{E}_{0} / \mathrm{S}_{0}\right)$. Los hidrolizados resultantes de la optimización fueron evaluados en sus propiedades antioxidantes con métodos in vitro, luego se sometieron a un proceso de digestión in vitro y se analizó su efecto sobre la capacidad antioxidante.

\section{MATERIALES Y MÉTODOS}

Se detalla la preparación de las muestras, los materiales y métodos usados, además del diseño de experimentos utilizado.

\section{Preparación de hidrolizados enzimáticos}

Se emplearon reactores de vidrio de $500 \mathrm{~mL}$ conectados a un regulador termostático. El control de pH se hizo empleando un titulador automático Titrando 842 (Metrohm, Suiza), operado por ordenador (software Tiamo 1.2.1). El medio de reacción fue constantemente agitado utilizando agitadores magnéticos (200 rpm). La sangre fue suministrada por Altecsan, una empresa dedicada a la comercialización de sangre higienizada, ubicada en la ciudad de Medellín-Colombia. Al momento de recepción del sustrato se realizaron controles microbiológicos de mohos y levaduras, coliformes totales y mesófilos. Las muestras de sangre bovina se centrifugaron a $4^{\circ} \mathrm{C}$ en una centrifuga Boeco (Ref. 1406-12) a 8500 rpm durante 10 min para separar la fracción globular (FG) del plasma, se realizó doble centrifugación para obtener la mayor recuperación de FG. Posteriormente la FG fue almacenada a $20{ }^{\circ} \mathrm{C}$ hasta el momento de la hidrólisis. El análisis del contenido proteico se llevó a cabo por el método de determinación del nitrógeno total Kjeldahl. La enzima usada fue Alcalase ${ }^{\circledR} 2,4 \mathrm{~L}$ grado alimenticio (Novozymes, Dinamarca), cuyo componente principal es Subtilisina A (Subtilisina Carlsberg). La concentración de enzima y el $\mathrm{pH}$ en cada ensayo se fijaron para cumplir con las relaciones enzima/sustrato $\left(\mathrm{E}_{0} / \mathrm{S}_{0}\right)$ que se definieron en el diseño experimental, de igual forma que el $\mathrm{pH}$, el cual se ajustó adicionando $\mathrm{NaOH} 1 \mathrm{~N}$ (preparado con $\mathrm{NaOH} 97 \%$ pureza Merck ${ }^{\circledR}$. Mientras que la temperatura y el tiempo de hidrólisis se fijaron en $55^{\circ} \mathrm{C}$ y $2 \mathrm{~h}$ respectivamente. 
La reacción fue monitoreada para la determinación del grado de hidrólisis $(G H)$, expresado como la relación entre el número de enlaces peptídicos cortados en la hidrólisis $(h)$ y el número de enlaces peptídicos totales en la proteína nativa por unidad de peso $\left(h_{t}\right)$. Para éste caso, se empleó un $h_{t}$ de 8,3 Eqv/Kg (Márquez y Vázquez, 1999). El método empleado para la determinación del grado de hidrólisis es el de valoración del protón o método del pH-estático Ecuaciones (1-3) (Adler-Nissen, 1986).

$$
\begin{aligned}
& G H=\frac{B N_{B}}{M_{p}} \frac{1}{\alpha} \frac{1}{h_{t}} 10 \\
& \alpha=\frac{10^{p H-p K}}{1+10^{p H-p K}} \\
& p k=7,8+\frac{298-T}{298 \times T} 2400
\end{aligned}
$$

Donde $B$ es el Volumen consumido de base en litros, $N_{B}=$ Normalidad de la base (Eqv/L), Mp es Masa de la proteína en $\mathrm{Kg}$ y a es el grado de disociación de los grupos a- $\mathrm{NH}_{2}$ liberados en la reacción, el cual depende del pK, que a su vez está asociado con la temperatura (Valencia et al., 2014).

\section{Ajuste experimental de datos}

Los valores del GH en el tiempo de reacción, obtenidos en cada corrida experimental se ajustaron al modelo exponencial propuesto por Márquez-Moreno y Fernández-Cuadrado (Márquez-Moreno y FernándezCuadrado, 1993; Qi y He, 2006) que permite analizar el comportamiento del GH en el tiempo con dos constantes cinéticas "a" y "b", como se indica en la Ecuación (4). Las constantes cinéticas se calcularon con los resultados de $\mathrm{GH}$ y tiempo, medidos cada cinco minutos hasta 2 horas, por el método de mínimos cuadrados utilizando la función "Isqcurvefit" del "toolbox" de Matlab 2014 R2014a. La bondad de ajuste del modelo se evaluó utilizando el coeficiente de determinación.

$$
G H=\frac{1}{b} \ln (a b t+1)
$$

\section{Determinación de la capacidad antioxidante}

Para la medición de la capacidad antioxidante se emplearon los métodos de determinación directa ABTS e indirecta ORAC y FRAP. Para el ajuste de las técnicas se usó un espectrofotómetro marca Thermo Scientific Genesis $10 \mathrm{~S}$ UV-vis. Para los tres métodos se establecieron curvas de calibración con diferentes concentraciones del antioxidante estándar Trolox (análogo hidrofílico del alfa tocoferol, Lote STBB72184, ALDRICH ${ }^{\circledR}$. Los resultados se expresan como micromoles de equivalentes Trolox por gramo de proteína ( $\mu \mathrm{molET} / \mathrm{g}$ proteína). Todos los reactivos usados para la medición de capacidades antioxidantes fueron de grado analítico.

\section{Método FRAP (Ferric reducing/antioxidant power)}

Mide el efecto que tienen los compuestos antioxidantes para reducir en un medio ácido el hierro férrico $\left(\mathrm{Fe}^{+3}\right)$ hasta la forma ferrosa $\left(\mathrm{Fe}^{+2}\right)$. Se siguió la metodología ajustada de Pulido et al., en la que $900 \mu \mathrm{L}$ del reactivo FRAP (con TPTZ, $\mathrm{FeCl}_{3}$ y buffer de acetato de sodio3 M, S7899 SIGMA ${ }^{\circledR}$ ) recién preparado y calentado a $37^{\circ}$ $\mathrm{C}$, se mezclan con $90 \mu \mathrm{L}$ de agua destilada y $30 \mu \mathrm{L}$ de la muestra o estándar Trolox y se incuban a $37^{\circ} \mathrm{C}$ por $30 \mathrm{~min}$; la absorbancia se lee a $595 \mathrm{~nm}$ (Pulido et al., 2000).

\section{Método TEAC (Trolox Equivalent Antioxidant Capacity) o ABTS}

Se fundamenta en la cuantificación de la decoloración del radical ABTS+ (ácido 2,2' azinobis-3etilbenzotiazolina-6- sulfonico) provocado por la interacción con moléculas donantes de electrones. Se basó en la metodología descrita por Re et al., en el cual $100 \mu \mathrm{L}$ de la muestra o estándar Trolox, son mezclados con $1 \mathrm{~mL}$ de la solución ABTS' e incubados a $30^{\circ} \mathrm{C}$ por $30 \mathrm{~min}$. Después de esto se toman las medidas de absorbancia a $730 \mathrm{~nm}$ (Re et al., 1999). 


\section{Método ORAC (Oxigen radical absorbance capacity)}

Mide el efecto estabilizador de los radicales libres que tienen los compuestos antioxidantes mediante un mecanismo de donación de un átomo de hidrógeno. La capacidad antioxidante del método Orac se determinó por la metodología descrita por Ou et al., en la mezcla de $150 \mu \mathrm{L}$ de flourescina $1 \mu \mathrm{M}$ (sustrato fluorescente) y $25 \mu \mathrm{L}$ de muestra (diluidos en buffer fosfato $10 \mu \mathrm{M}$ a pH 7,4 hasta una concentración de proteínas de 0,2 $\mathrm{mg} / \mathrm{mL}$ ), fueron preincubada por $30 \mathrm{~min}$ a $37^{\circ} \mathrm{C}$. Luego $25 \mu \mathrm{L}$ de una solución de 2,2'-azobis (2methylpropionamidine) dihydrochloride (AAPH) $250 \mathrm{mM}$ fue adicionada. La intensidad de la fluorescencia fue medida con longitudes de onda de excitación y emisiones fijadas en 485 y $520 \mathrm{~nm}$, respectivamente (Ou et al., 2001).

\section{Digestión in vitro}

Tanto a la FG entera como a la hidrolizada se les realizó un ensayo de digestión in vitro, siguiendo la descripción de Gómez et al., 2013, que fue ajustada de la metodología de Yagoub et al., 2004, en la cual la muestra de interés fue incubada con $10 \mu \mathrm{g}$ de pepsina/mg proteína (E.C. 3.4.23.1; $\geq 250 \mathrm{U} \mathrm{mg}^{-1}$ sólido; Sigma, Louis, USA), a un pH de 2 ajustado con $\mathrm{HCl}$, durante $3 \mathrm{~h}$ a $37^{\circ} \mathrm{C}$, se ajustó el pH de la mezcla entre 7,5 a 8,0 con adición de $\mathrm{NaOH}(0,2 \mathrm{~N})$, luego la adición del buffer fosfato $(\mathrm{pH} 7.4)$ con $1 \mathrm{mM}$ de $\mathrm{CaCl}, 0,01 \%$ de $\mathrm{NaN}_{3}$ y $13 \mu \mathrm{g}$ de pancreatina/mg de proteína (Sigma, No. P1750). Finalmente la mezcla se incubó a $37^{\circ} \mathrm{C}$ durante 24 horas (Gomez et al., 2013; Yagoub et al., 2004). Los hidrolizados se inactivaron y almacenaron a $-20^{\circ} \mathrm{C}$ para la determinación de la capacidad antioxidante.

\section{Diseño experimental}

Se analizó la influencia de los factores $\mathrm{pH}$, concentración inicial de sustrato $\left(\mathrm{S}_{0} \mathrm{~m} / \mathrm{V}\right)$ y relación enzima/sustrato $\left(E_{0} / S_{0} m / m\right)$, sobre las variables respuesta, las cuales fueron el $G H$ y las constantes cinéticas a y b de la Ecuación (4). Para ello se ejecutó un diseño de experimentos Central Compuesto Rotable, con los niveles de los factores que se indican en la Tabla 1. El análisis de varianza (ANOVA) y el ajuste de los modelos (Ecuación 5), se hicieron utilizando el software Statgraphics Centurion XVI. La calidad de los ajuste se verificó por medio del $R^{2}$ y el $R^{2}$ Ajustado. La significancia de los coeficientes estimados en los modelos se probó con el estadístico $\mathrm{F}$ a un nivel de confianza de $95 \%$ (valor $\mathrm{p}<0,05$ ). Una vez obtenido el modelo, se empleó la metodología de superficie de respuesta MSR para establecer las condiciones óptimas de trabajo recomendadas para maximizar el rendimiento de la hidrólisis (maximizar el GH).

Tabla1: Variables independientes y sus niveles codificados y reales utilizados en el diseño MSR

\begin{tabular}{lccccc}
\hline \multirow{2}{*}{ Variable } & & \multicolumn{4}{c}{ Niveles codificados } \\
\cline { 3 - 6 } & $-1,68$ & -1 & 0 & +1 & 1,68 \\
\hline $\mathrm{pH}$ & 7,32 & 8 & 9 & 10 & 10,68 \\
$\mathrm{~S}_{0}(\% \mathrm{~m} / \mathrm{v})$ & 6,63 & 8 & 10 & 12 & 13,36 \\
$\mathrm{E}_{0} / \mathrm{S}_{0}(\% \mathrm{~m} / \mathrm{m})$ & 1,27 & 4 & 8 & 12 & 14,73 \\
\hline
\end{tabular}

$$
Y_{j}=\beta_{0}+\sum \beta_{i} x_{i}+\sum \beta_{i i} x_{i}^{2}+\sum \beta_{i j} x_{i} x_{j}
$$

Donde $Y_{j}$ es la respuesta predicha $(\mathrm{GH}), \mathrm{X}_{i}$ y $\mathrm{X}_{j}$ desde $i, j=1$ hasta 3 son las variables independientes o factores; $\beta_{0}, \beta_{i}, \beta_{i i}, \beta_{i j}$, son los términos independientes, lineales, cuadráticos y de interacción del polinomio.

En el caso de los resultados de análisis de capacidad antioxidante por los métodos FRAP, ABTS y ORAC se hicieron por triplicado y se realizó un análisis de diferencias significativas entre medias con el test de rangos múltiples de Duncan. La significancia de las comparaciones entre medias de la capacidad antioxidantes se probó con el estadístico $F$ y un nivel de confianza del $95 \%(P<0,05)$.

\section{Optimización por MSR}

Una de las herramientas generalmente empleadas para establecer condiciones óptimas de operación es la metodología de superficie de respuesta, la cual se basa en el método de la máxima pendiente en ascenso, que consiste en recorrer la trayectoria de máxima pendiente, que conduce a la dirección del máximo cambio en la respuesta (Montgomery, 2013). La región de exploración son los niveles codificados de los factores evaluados. En este estudio se empleó el software Statgraphics Centurion ${ }^{\circledR}$, para corroborar los resultados de la rutina de optimización. Los valores óptimos de los factores $\left(\mathrm{pH}, \mathrm{S}_{0}\right.$ y $\left.\mathrm{E}_{0} / \mathrm{S}_{0}\right)$, se emplearon en la ejecución de tres replicas experimentales, cuyos resultados se compararon con los predichos por el modelo polinomial ajustado. En estos ensayos se tomaron muestras a diferentes tiempos de hidrólisis para someterlas al proceso de digestión in vitro y posteriormente determinarles las capacidades antioxidantes (FRAP y ABTS) antes y después de la digestión. 


\section{RESULTADOS Y DISCUSIÓN}

Se describen los resultados en subsecciones para una mejor organización de los resultados presentados con su discusión.

\section{Determinación de las constantes de la Ecuación de Hidrólisis enzimática de la FG de sangre bovina}

Los valores del GH para la hidrólisis enzimática de la FG y las constantes "a" y "b" estimadas por regresión no lineal de la Ecuación (4), así como los coeficientes de determinación $\left(R^{2}\right)$, se muestran en la Tabla 2. En la cual se evidenció la relación entre las variables independientes codificadas y el rendimiento de la reacción (GH) para las 19 corridas definidas por el diseño experimental.

Tabla 2: Diseño central compuesto para la hidrólisis enzimática de la fracción globular de sangre bovina. Valores de $\mathrm{GH}$, a y b, obtenidos de la curva de hidrolisis a 2 horas de reacción.

\begin{tabular}{|c|c|c|c|c|c|c|c|}
\hline \multirow{2}{*}{$N^{\circ}$ corrida } & $p H$ & So & $E_{0} / S_{0}$ & \multirow{2}{*}{$G H$} & \multirow{2}{*}{$a$} & \multirow{2}{*}{$B$} & \multirow{2}{*}{$R^{2}$} \\
\hline & \multicolumn{3}{|c|}{ Variables codificadas } & & & & \\
\hline 1 & -1 & 1 & 1 & 27,0752 & 6,7784 & 0,1718 & 0,957 \\
\hline 2 & 0 & 0 & 0 & 30,5763 & 14,8029 & 0,1835 & 0,9682 \\
\hline 3 & 0 & $-1,68$ & 0 & 29,0578 & 15,1328 & 0,1897 & 0,9533 \\
\hline 4 & 0 & -1 & -1 & 23,1937 & 2,3383 & 0,1546 & 0,9178 \\
\hline 5 & 1 & -1 & -1 & 19,1689 & 4,6055 & 0,2494 & 0,9926 \\
\hline 6 & 1 & 1 & -1 & 27,8498 & 1,7835 & 0,1104 & 0,9555 \\
\hline 7 & -1 & -1 & 1 & 31,7236 & 9,0767 & 0,1528 & 0,9579 \\
\hline 8 & 0 & 1,68 & 0 & 31,0637 & 5,5459 & 0,1376 & 0,9358 \\
\hline 9 & 1 & 1 & 1 & 26,0182 & 5,0398 & 0,1736 & 0,9678 \\
\hline 10 & 1,68 & 0 & 0 & 22,5548 & 32,7888 & 0,2942 & 0,9547 \\
\hline 11 & 0 & 0 & 0 & 31,2958 & 8,2872 & 0,1531 & 0,9636 \\
\hline 12 & 0 & 0 & 1,68 & 34,2958 & 17,8838 & 0,1572 & 0,9555 \\
\hline 13 & $-1,68$ & 0 & 0 & 22,7574 & 2,0376 & 0,1496 & 0,9708 \\
\hline 14 & 0 & 0 & 0 & 31,0476 & 11,4581 & 0,1698 & 0,9836 \\
\hline 15 & 0 & 0 & 0 & 30,3410 & 8,1447 & 0,1605 & 0,9866 \\
\hline 16 & 1 & -1 & 1 & 25,3664 & 43,4013 & 0,2667 & 0,9587 \\
\hline 17 & 0 & 0 & $-1,68$ & 21,5955 & 1,433 & 0,1492 & 0,9991 \\
\hline 18 & -1 & 1 & -1 & 22,6949 & 3,1362 & 0,1841 & 0,9979 \\
\hline 19 & 0 & 0 & 0 & 28,1226 & 7,0187 & 0,1714 & 0,9925 \\
\hline
\end{tabular}

Se obtuvo un buen ajuste de las constantes cinéticas "a" y "b" para la mayoría de las reacciones de hidrólisis experimentales del diseño $\left(R^{2}>0,95\right)$. Esta calidad del ajuste de la Ecuación (4) ha sido reportada por diferentes autores para otros sustratos (Valencia et al., 2014; Valencia et al., 2015; Apar y Özbek, 2008; Ruan et al., 2010; Demirhan et al., 2011).

\section{Efectos de los factores sobre las respuestas}

En la Tabla 3 se muestran los resultados del análisis de varianza (ANOVA) para la prueba de significancia de los coeficientes del modelo. El análisis indica que $\mathrm{E}_{0} / \mathrm{S}_{0}$ es el factor más significativo sobre el GH, puesto que los términos lineales y cuadráticos muestran un efecto muy significativo elevado con valores $p \leq 0,01$ y $p \leq$ 0,05 respectivamente.

En la región de exploración los efectos lineales de $\mathrm{S}_{0}$ y el pH no resultan significativos, sin embargo las interacciones de estos factores si lo son $(p \leq 0,05)$ y a juzgar por el considerable valor del coeficiente en el modelo, estas dos variables juegan un papel importante en la predicción del GH. Teniendo en cuenta que el $\mathrm{pH}$ tiene un efecto cuadrático elevado con $\mathrm{p} \leq 0,01$, es concluyente que este factor presenta un efecto dual sobre la respuesta $(\mathrm{GH})$ en la región de exploración, indicando que aunque la enzima utilizada es de amplio rango de trabajo a $\mathrm{pH}$ alcalino con este sustrato (hemoglobina bovina), lo más recomendable es operar a valores de $\mathrm{pH}$ cercanos a 8,0. La actividad de la enzima se ve fuertemente afectada por el $\mathrm{pH}$, debido a que el estado activo de la enzima se presenta en alguna forma ionizada de la misma, lo cual está influenciado por 
los cambios de pH y la disociación de los grupos activos, propiciando mayor afinidad por el sustrato (Shi et al., 2005). Este fenómeno explica entre otras cosas la significancia estadística de la interacción $\mathrm{pH}^{*} \mathrm{~S}_{0}$ del análisis mostrado (Tabla 3). Los resultados muestran además que la disminución del rendimiento de la reacción a valores de $\mathrm{pH}$ elevados, puede deberse a fenómenos de desnaturalización de la enzima a esos valores de $\mathrm{pH}$.

Tabla 3: Significancia para los coeficientes de regresión del modelo ajustado.

\begin{tabular}{|c|c|c|c|c|c|c|}
\hline \multirow{2}{*}{ Fuente } & \multicolumn{2}{|c|}{$\mathrm{GH}$} & \multicolumn{2}{|c|}{ "a" } & \multicolumn{2}{|c|}{ "b" } \\
\hline & Coeficiente & Valor-P & Coeficiente & Valor-P & Coeficiente & Valor-P \\
\hline \multicolumn{7}{|l|}{ Lineal } \\
\hline$p H$ & $-0,3011$ & 0,4620 & 7,5400 & 0,0012 & 0,0296 & 0,0003 \\
\hline So & 0,7409 & 0,1018 & $-3,2302$ & 0,0205 & $-0,01644$ & 0,0101 \\
\hline$E_{0} / S_{0}$ & 3,0164 & 0,0010 & 6,9410 & 0,0013 & - & 0,0019 \\
\hline \multicolumn{7}{|l|}{ Cuadrático } \\
\hline$p H^{2}$ & $-2,9736$ & 0,0009 & - & 0,0026 & 0,0211 & \\
\hline$\left(E_{0} / S_{0}\right)^{2}$ & $-1,1064$ & 0,0312 & - & & - & \\
\hline \multicolumn{7}{|l|}{ Interacciones } \\
\hline$p H^{*} S_{0}$ & 1,4958 & 0,0425 & $-7,1623$ & 0,0045 & $-0,0381$ & 0,0005 \\
\hline$p H^{*} E_{0} / S_{0}$ & $-1,3822$ & 0,0533 & - & - & - & \\
\hline$S_{0}{ }^{*} E_{0} / S_{0}$ & $-1,8422$ & 0,0167 & $-6,6660$ & 0,0043 & - & \\
\hline Falta de ajuste & & 0,2945 & & 0,1289 & & 0,2612 \\
\hline Total (corr.) & & 19 & & & & \\
\hline
\end{tabular}

El análisis de los efectos de las variables independientes sobre las constantes cinéticas "a" y "b" del sistema de reacción Alcalasa-FG de sangra bovina, se realizó siguiendo la metodología propuesta por Valencia $\mathrm{P}$, et al, la cual analiza las constantes cinéticas como función de las condiciones de operación de la reacción (Valencia et al., 2015). Los parámetros cinéticos estimados por el ajuste de la Ecuación (4) empleando datos experimentales se muestran en la Tabla 3. Los resultados son coherentes con el argumento y la expresión de los mecanismos desarrollados expuesto por Qi et al., 2006, quienes al deducir las posibles expresiones de los parámetros en la Ecuación (4) de acuerdo con el mecanismo cinético asumido, indican que a pesar de los posibles mecanismos expuestos, el parámetro "a" siempre depende de la concentración de enzima ( $\left.E_{0}\right)$ y el parámetro "b" de la constante cinética $\left(K_{m}\right)$ (Qi y He, 2006). Los resultados muestran que los valores de "a" efectivamente aumentan con la $\mathrm{E}_{0} / \mathrm{S}_{0}$, cuando la temperatura y $\mathrm{pH}$ permanecen constantes, lo cual es consistente con los resultados de varios autores (Figueroa et al., 2012; Valencia et al., 2015; Camacho et al., 1993).

Los valores de "b" decrecen cuando la hidrólisis ocurre en concentraciones de sustrato elevadas, lo cual se detecta por el coeficiente negativo para la concentración de sustrato en la Ecuación (8), con valor $p<0,05$. Sin embargo no existe relación estadística significativa entre estos valores y el $\mathrm{GH}$. En términos generales existe una fuerte tendencia a que los valores más bajos de "b" se registran cuando mayor es el GH, lo que sin duda indicaría mayor afinidad de la Alcalasa 2,4 L con la fracción globular de sangre bovina, independientemente del mecanismo cinético expuesto. Para soportar estadísticamente esta afirmación se sugiere disminuir el error en la determinación de los parámetros de ajuste, utilizando un mayor número de puntos en cada curva de hidrólisis. La constante "a” presenta relación estadística significativa con el pH y la $E_{0} / S_{0}$, pues se reportan importantes incrementos de la velocidad inicial de hidrólisis en la medida en que estas variables aumentan. Valencia et al, sugieren que las constantes cinéticas "a" y "b" se pueden utilizar para comparar y caracterizar la eficacia de la proteasa y la susceptibilidad a la hidrólisis por parte de los sustratos (Valencia et al., 2015).

Los modelos estadísticos ajustados con sus coeficientes calculados por regresión múltiple, se muestran en las Ecuaciones (6-8). Estos modelos son resultado de la exclusión de términos no significativos $(p>0,05)$, conservando en cada caso la jerarquía de los mismos.

$$
\begin{aligned}
& G H=29,95-0,30 p H+0,74 S_{0}+3,02 E / S_{0}-2,97 p H^{2}+1,5 p H * S_{0}-1,38 p H * E_{0}-1,84 S_{0} * E / S_{0}-1,11 E / S_{0}^{2} \\
& \mathrm{a}=9,79+7,53 \mathrm{pH}-3,23 \mathrm{~S}_{0}+6,94 E / S_{0}-7,16 \mathrm{pH} * \mathrm{~S}_{0}-6,67 \mathrm{~S}_{0} * E / S_{0} \\
& \mathrm{~b}=0,16+0,03 \mathrm{pH}-0,02 \mathrm{~S}_{0}+0,02 p H^{2}-0,04 p H * S_{0}
\end{aligned}
$$




\section{Maximización del GH para la hidrólisis enzimática del FG bovina}

El modelo de la Ecuación (6) se sometió a un proceso de optimización, para maximizar el GH. En la Tabla 4, se muestran los resultados de la optimización empleando MSR, aquí se registran los valores óptimos de los factores evaluados (codificados y reales) y el GH (variable respuesta) predicho frente al valor experimental obtenido. La Figura 1, muestra el gráfico de superficie de respuesta del (GH) en función de los factores más significativos en el estudio ( $\mathrm{pH}$ y $\mathrm{E}_{0} / \mathrm{S}_{0}$ ). En este caso la relación concentración de sustrato ( $\mathrm{S}_{0}$ ) se fijó en su máximo nivel codificado, mientras que el $\mathrm{pH}$ y $\mathrm{E}_{0} / \mathrm{S}_{0}$ se establecieron en los ejes $\mathrm{X}$ y $\mathrm{Y}$ de la gráfica. El GH corresponde a una superficie curva, debido a la importancia de los efectos de segundo grado. La optimización numérica de $\mathrm{GH}$, por MSR entregó un valor de $38 \%$, para condiciones de $\mathrm{pH}=8,14 ; \mathrm{S}_{0}=6,64$ y $\mathrm{E}_{0} / \mathrm{S}_{0}=14,73$.

Para confirmar la validez del modelo estadístico propuesto, se llevaron a cabo tres corridas experimentales bajo las condiciones óptimas establecidas, encontrando que el GH experimental fue de 36,25 \pm 0,31\%, lo que representa un sesgo negativo del 5,03 \% en relación con el GH esperado. La prueba de falta de ajuste con p>0,05 y la buena precisión de los experimentos sugiere que efectivamente los valores encontrados de los niveles para cada factor evaluado, son condiciones óptimas de trabajo para este sistema de reacción.

Tabla 4: Condiciones óptimas de los factores experimentales en la hidrolisis enzimática de la FG bovina.

\begin{tabular}{l|l|l|l|l}
\hline \multicolumn{2}{c|}{ Condiciones Optimas } & \multicolumn{2}{c}{ GH (\%) } \\
\hline \multirow{2}{*}{ Variables } & $\begin{array}{l}\text { Valor Codificado con } \\
\text { MSR }\end{array}$ & $\begin{array}{l}\text { Valor sin Codificar con } \\
\text { MSR }\end{array}$ & Predicho & \multirow{2}{*}{ Experimental } \\
\hline$p H$ & $-0,8643$ & 8,1 & & \\
\hline$S_{0}(\mathrm{~m} / \mathrm{v})$ & $-1,68$ & 6,6 & \multirow{3}{*}{38,08} & \multirow{2}{*}{36,25} \\
\hline$E_{0} / S_{0}(\mathrm{~m} / \mathrm{m})$ & 1,68 & 14,7 & & \\
\hline
\end{tabular}

En lo que respecta al $\mathrm{GH}$, el análisis de varianza (ANOVA) demuestra que el modelo representa adecuadamente los datos experimentales, teniendo en cuenta que el $R^{2}$ es de 0,9264. Lo que indica que éste describe la influencia de las variables estudiadas sobre el GH de las proteínas de la fracción globular de sangre bovina en un porcentaje satisfactorio de $93 \%$ aproximadamente. El estadístico $\mathrm{R}^{2}$ ajustado es de 87 $\%$, lo cual nos dice que efectivamente los factores estudiados, junto con sus interacciones explican en un porcentaje satisfactorio la variabilidad observada en el $\mathrm{GH}$, por tanto, el modelo propuesto es una buena descripción del fenómeno de hidrólisis enzimática de este sustrato con Alcalasa 2,4 L.

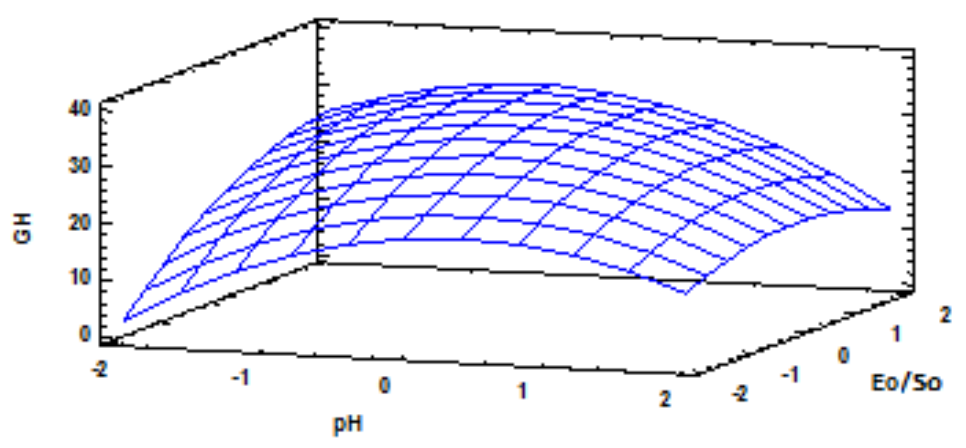

Fig. 1: Superficie de respuesta para GH con So constante $10 \%(\mathrm{~m} / \mathrm{V})$.

Estos resultados muestran, que es importante analizar el efecto del pH y la concentración de sustrato sobre el comportamiento cinético en reacciones de hidrólisis de proteínas, pues estas condiciones ofrecen información de gran valor en el análisis del rendimiento de estas reacciones, lo cual permite avanzar en criterios de identificación de condiciones de trabajo ideales para la identificación de fracciones peptídicas de interés bioactivo.

\section{Capacidad antioxidante}

Se determinaron las capacidades antioxidantes de la FG y sus hidrolizados en las condiciones óptimas del diseño a diferentes tiempos de hidrólisis, además se simuló la digestión in vitro para ver el efecto que tiene sobre la capacidad antioxidante. Los resultados con sus respectivas desviaciones se muestran en la Tabla 5, los valores están dados en $\mu \mathrm{mol}$ ET/g proteína. Las medias (3 réplicas) de la misma columna con diferente superíndice difieren significativamente $(p<0,05)$. 
Tabla 5: Capacidades antioxidantes con los métodos ORAC, FRAP y ABTS de la FG de sangre bovina, sus hidrolizados antes y después de digestión in vitro a diferentes tiempos de hidrólisis.

\begin{tabular}{lccr}
\hline Tipo Muestra & ORAC & \multicolumn{1}{l}{ FRAP } & \multicolumn{1}{c}{ ABTS } \\
\hline FGSH & - & $45,2 \pm 2,5^{\mathrm{a}}$ & $608,9 \pm 4,1^{\mathrm{a}}$ \\
FGPD & - & $45,7 \pm 1,8^{\mathrm{a}}$ & $1038,22 \pm 9,5^{\mathrm{c}}$ \\
FG30SD & $461,2 \pm 37,7^{\mathrm{a}}$ & $54,8 \pm 2,2^{\mathrm{b}}$ & $747,5 \pm 68,5^{\mathrm{b}}$ \\
FG30PD & - & $81,8 \pm 5,1^{\mathrm{d}}$ & $1417,75 \pm 4,1^{\mathrm{e}}$ \\
FG60SD & $464,2 \pm 14,0^{\mathrm{a}}$ & $57,4 \pm 1,8^{\mathrm{b}}$ & $725,6 \pm 56,0^{\mathrm{b}}$ \\
FG60PD & - & $70,4 \pm 2,1^{\mathrm{c}}$ & $1396 \pm 6,3^{\mathrm{e}}$ \\
FG120SD & $513,7 \pm 8,2^{\mathrm{b}}$ & $65,3 \pm 5,8^{\mathrm{c}}$ & $748,3 \pm 71,3^{\mathrm{b}}$ \\
FG120PD & - & $64,8 \pm 2,4^{\mathrm{c}}$ & $1245,6 \pm 7,2^{\mathrm{d}}$ \\
\hline
\end{tabular}

En las actividades antioxidantes por lo métodos ORAC y FRAP existieron diferencias significativas entre los hidrolizados sin digestión a diferentes tiempos $(p<0,05)$. Mientras en el análisis de ABTS los hidrolizados sin digestión no difieren entre ellos $(p<0,05)$. Esto quiere decir que para obtener la mayor capacidad de reducción de radicales libres de Oxigeno y Fe se debe efectuar a un tiempo de hidrólisis de 2 horas bajo las condiciones óptimas, mientras que para obtener la máxima capacidad de los agentes antioxidantes de los hidrolizados de FG de sangre bovina para atacar el catión radical $\mathrm{ABTS}^{+}$basta con ejecutar la hidrólisis enzimática a 30 min, o cualquier otro tiempo estudiado.

Es claro que no es posible hacer comparaciones ni equivalencias entre los distintos métodos que analizan la actividad antioxidante, debido a los diversos mecanismos de acción de los antioxidantes de origen biológico que involucran procesos químicos variados (Prior, 2015). De igual manera, existen diferentes metodologías y/o diferentes unidades de expresión de capacidad antioxidante, lo que dificulta las comparaciones entre diferentes reportes de literatura (Samaranayaka y Li-Chan, 2011). Se ha evidenciado que la capacidad antioxidante de alimentos provenientes de fuentes proteicas independientemente de la metodología, son menos propensos a la capacidad de reducción del ion $\mathrm{Fe}^{+3}$, que a la capacidad de captación de radicales ABTS y peroxilo (Gomez et al., 2013), como efectivamente se comprobó en este estudio, puesto que a pH acido $(3,6)$, los registros de actividad antioxidante empleando el método FRAP son sustancialmente bajos. Aquí se debe tener en cuenta que el ensayo FRAP es diferente de los ensayos ORAC y ABTS porque no hay radicales libres u oxidantes implicados (Cao y Prior, 1998).

La digestión in vitro tuvo un efecto significativo favorable para la captación de radicales ABTS por parte de los hidrolizados, mientras que no afectó la captación de los mismos radicales por parte de la FG sin hidrolizar. Así mismo, se favoreció la capacidad de reducción del ion $\mathrm{Fe}^{+3}$, tanto para la fracción globular sin hidrolizar como para sus hidrolizados a diferentes tiempos de hidrolisis, posiblemente porque durante el proceso de digestión in vitro se liberan péptidos con capacidad antioxidante (Zhu et al., 2008; Borawska et al., 2016).

\section{CONCLUSIONES}

(i) La hidrólisis enzimática $(\mathrm{GH})$ de proteínas de la fracción celular de la sangre de bovino con la enzima Alcalasa $2,4 \mathrm{~L}$ se ve afectada por el pH, la concentración inicial de sustrato y la relación enzima sustrato, llegando al máximo $\mathrm{GH}$ cuando se trabaja $\mathrm{pH}: 8,16 ; \mathrm{S}: 6,6$ y $\mathrm{E}_{0} / \mathrm{S}_{0}: 14,7$. (ii) La actividad antioxidante de los hidrolizados de la fracción celular de la sangre depende del tiempo de hidrolisis, siendo el tiempo de $2 \mathrm{~h}$ el que mayores actividades antioxidantes genera. (iii) La digestión in vitro puede favorecer la actividad antioxidante de los hidrolizados de la fracción celular de sangre bovina.

\section{AGRADECIMIENTOS}

A la Universidad Popular del Cesar, por la financiación del proyecto de investigación y a Colciencias por la financiación en conjunto con la misma Universidad de una beca-pasantía a través del programa de Jóvenes Investigadores e Innovadores. Así mismo a la Universidad de Antioquia y a su programa de sostenibilidad.

\section{NOTACIÓN}

FG

$\mathrm{GH}$

FGSH

FGPD

FG30SD

FG30PD

FG60SD

FG60PD

FG120SD

FG120PD MSR
Fracción globular

Grado de hidrólisis

Fracción globular bovina sin hidrolizar

Fracción globular bovina con digestión

Hidrolizado de Fracción globular bovina 30 min sin digestión

Hidrolizado de Fracción globular bovina 30 min por digestión

Hidrolizado de Fracción globular bovina 60 min sin digestión

Hidrolizado de Fracción globular bovina 60 min por digestión

Hidrolizado de Fracción globular bovina 120 min sin digestión

Hidrolizado de Fracción globular bovina 120 min por digestión

Metodología superficie de respuesta 


\section{REFERENCIAS}

Adler-Nissen, J., Enzymic hydrolysis of food proteins, 116-124, Elsevier, Londres, Inglaterra (1986)

Apak, R., M. Özyürek, K. Güçlü y E. Çapanoğlu, Antioxidant Activity/Capacity Measurement. 1. Classification, Physicochemical Principles, Mechanisms, and Electron Transfer (ET)-Based Assays, doi: 10.1021/acs.jafc.5b04739, Journal of Agriculture and Food Chemistry (en línea), 64 (5), 997-1027 (2016)

Apar, D. y Özbek, B., Corn gluten hydrolysis by Alcalase: effects of process parameters on hydrolysis, solubilization and enzyme inactivation, Chemical and Biochemical Engineering Quarterly, ISSN: 0352-9568 (en línea), 22(2), 203-212, http://silverstripe.fkit.hr/cabeq/assets/Uploads/Cabeq-2008-02-09.pdf (2008)

Bah, C., Bekhit, A., Carne, A. y McConnell, M., Slaughterhouse blood: An emerging source of bioactive compounds, doi: 10.1111/1541-4337.12013, Comprehensive Review Food Science and Food Safety (en línea), 12 (3), 314-331 (2013)

Borawska, J., Darewicz, M., Pliszka, M. y Vegarud, G., Antioxidant properties of salmon (Salmo salar L.) protein fraction hydrolysates revealed following their ex vivo digestion and in vitro hydrolysis, doi: 10.1002/jsfa.7441, Journal of the Science of Food and Agriculture (en línea), 96(8), 2764-2772 (2016)

Camacho, F., González, P., Páez, M., Márquez, M.C., Fernández, V., Hidrólisis de caseína con alcalasa, Revista Española de Ciencia y Tecnología de Alimentos, ISSN: 1131-799X (en línea), 33(1), 59-70, http://www.ugr.es/ fcamacho/Originales/Trabajos\%20Publicados/RECTA1993.pdf (1993)

Cao, G. y Prior, R. Comparison of different analytical methods for assessing total antioxidant capacity of human serum., Clinical Chemistry, ISSSN: 1309-1315 (en línea), 44(6), 1309-1315, http://clinchem.aaccjnls.org/content/clinchem/44/6/1309.full.pdf (1998)

Chang, Chi-Yue., Kuei-Ching, W. y Shu-Hua C., Antioxidant Properties and Protein Compositions of Porcine Haemoglobin Hydrolysates, doi: 10.1016/j.foodchem.2005.12.019, Food Chemistry (en línea), 100(4),1537$1543(2007)$

De Castro, R. J. S. y Sato, H.H., Biologically active peptides: Processes for their generation, purification and identification and applications as natural additives in the food and pharmaceutical industries, doi:10.1016/j.foodres.2015.05.013, Food Research International, 74, 185-198 (2015)

Demirhan, E., Apar, D. y Özbek, B., Sesame cake protein hydrolysis by Alcalase: effect of process parameters on hydrolysis, solubilization and enzyme inactivation, doi: 10.1007/s11814-010-0316-2, Korean Journal of Chemical Engineering (en línea), 28(1), 195-202 (2011)

Figueroa, O., Zapata, J. y Gutierrez, G. Modelamiento de la cinética de hidrólisis enzimática de proteínas del plasma bovino, Revista EIA, ISSN: 1794-1237 (en línea), 9(17), 71 - 84, http://revistabme.eia.edu.co/index.php/reveia/article/view/452/444 (2012)

Figueroa, O., Zapata, J. y Sánchez, C., Optimización de La Hidrólisis Enzimática de Proteínas de Plasma Bovino, doi: 10.4067/S0718-07642016000200006, Información Tecnológica (en línea), 27(2), 39-52 (2016)

Gomes, I., Dale, C., Casten, K., Geigner, M., Gozzo, F., Ferro, E., Heimann, A y Devi, L., Hemoglobin-derived Peptides as Novel Type of Bioactive Signaling Molecules, doi: 10.1208/s12248-010-9217-x, American Association of Pharmaceutical Scientists Journal (en línea), 12(4), 658-669 (2010)

Gomez, L., Figueroa, O. y Zapata, J., Actividad Antioxidante de Hidrolizados Enzimáticos de Plasma Bovino Obtenidos por Efecto de Alcalasa® 2.4 L., doi: 10.4067/S0718-07642013000100005, Información Tecnológica (en línea), 24(1), 33-42 (2013)

Laca, A., Diaz, M. y Rendueles, M., Alternativas e implicaciones medioambientales de la gestión de residuos en la industria cárnica, Alimentación, Equipos y Tecnología, ISSN: ISSN 0212-1689 (en línea), 23 (189), $92-$ 99, https://dialnet.unirioja.es/servlet/articulo?codigo=859857 (2004)

Liu, Q., Kong, B., Xiong, Y. y Xia, X., Antioxidant activity and functional properties of porcine plasma protein hydrolysate as influenced by the degree of hydrolysis., doi: 10.1016/j.foodchem.2009.05.013, Food Chemistry (en línea), 118(2), 403-410 (2010) 
Liu, Y., L. Xianghong y otros cuatro autores, Characterization of structural and functional properties of fish protein hydrolysates from surimi processing by-products, doi:10.1016/j.foodchem.2013.11.089, Food Chemistry (en línea), 151(15), 459-465 (2014)

Márquez-Moreno, M. y Fernández-Cuadrado, V., Enzymic hydrolysis of vegetableproteins: mechanism and kinetics., doi: 10.1002/bit.260220804, Process Biochem (en línea), 28(7), 481-490 (1993)

Márquez, M. y Vázquez, M., Modeling of enzymatic protein hydrolysis., doi: 10.1016/S0032-9592(99)000412, Process Biochemistry (en línea), 35 (1), 111-117 (1999)

Möller, N., Scholz-Ahrens, K., Roos, N. y Schrezenmeir, J., Bioactive peptides and proteins from foods: Indication for health effects, doi: 10.1007/s00394-008-0710-2, European Journal of Nutrition (en línea), 47(4), 171-182(2008)

Montgomery, D., Design and Analysis of Experiments, $8^{\text {th }}$ edition, 478-544, John Wiley \& Sons, Inc., Hoboken, NJ, USA (2013)

Opheim, M., R. Šližytėb y otros cuatro autores, Hydrolysis of Atlantic salmon (Salmo Salar) rest raw materialsEffect of raw material and processing on composition, nutritional value, and potential bioactive peptides in the hydrolysates, doi:10.1016/j.procbio.2015.04.017, Process Biochemistry (en línea), 50(8), 1247-1257 (2015)

Ou, B., Hampsche-Woodill, M. y Prior, R., Development and validation of an improved oxygen radical absorbance capacity assay using fluorescein as the fluorescent probe, doi: 10.1021/jf010586o, Journal of Agricultural and Food Chemistry (en línea), 49 (10), 4619-4626 (2001)

Prior, R., Oxygen radical absorbance capacity (ORAC): New horizons in relating dietary antioxidants/bioactives and health benefits, doi: 10.1016/j.jff.2014.12.018, Journal of Functional Foods (en línea), 18(B), 797-810 (2015)

Pulido, R., Bravo, L. y Saura-Calixto, F., Antioxidant activity of dietary polyphenols as determined by a modified ferric reducing/antioxidant power assay, doi: 10.1021/jf9913458, Journal of Agricultural and Food Chemistry (en línea), 48(8), 3396-3402 (2000)

Qi, W. y He, Z., Enzymatic hydrolysis of protein: Mechanism and kinetic model, doi: 10.1007/s11458-0060026-9, Frontiers of Chemistry in China (en línea), 1(3), 308-314 (2006)

Re, R., Pellegrini, N., Proteggente, A., Pannala, A., Yang, M., Rice-Evans, C., Antioxidant activity applying an improved ABTS radical cation decolorization assay, doi: 10.1016/S0891-5849(98)00315-3, Free Radical Biology y Medicine (en línea), 26(9), 1231-1237 (1999)

Ruan, C., Chi, Y. y Zhang, R., Kinetics of hydrolysis of egg white protein by pepsin, Czech Journal of Food Sciences, ISSN: 1805-9317, 28(5), 355-363 (2010)

Saidi, S., Deratani, A., Ben Amar, R. y Belleville, M.P., Fractionation of a tuna dark muscle hydrolysate by a two-step membrane process, doi:10.1016/j.seppur.2013.01.048, Separation and Purification Technology (en línea), 108, 28-36 (2013)

Saidi, S., Deratani, A., Belleville, M.P. y Amar, R.B., Production and fractionation of tuna by-product protein hydrolysate by ultrafiltration and nanofiltration: Impact on interesting peptides fractions and nutritional properties, doi:10.1016/j.foodres.2014.04.026, Food Research International, 65(Part C), 453-461 (2014)

Samaranayaka, A. y Li-Chan, E., Food-derived peptidic antioxidants: A review of their production, assessment, and potential applications., doi: 10.1016/j.jf.2011.05.006, Journal of Functional Foods (en línea), 3(4), 229$254(2011)$

Shi, D., He, Z. y Qi, W., Lumping kinetic study on the process of tryptic hydrolysis of bovine serum albumin., doi: 10.1007/s11458-006-0026-9, Process Biochemistry (en línea), 40(5), 1943-1949 (2005)

Tavano, O., Protein hydrolysis using proteases: An important tool for food biotechnology., doi: 10.1016/j.molcatb.2013.01.011, Journal of Molecular Catalysis B: Enzymatic (en línea), 90, 1-11 (2013) 
Valencia, P., Espinoza, K., Ceballos, A., Pinto, M., Almonacid, S., Novel modeling methodology for the characterization of enzymatic hydrolysis of proteins, doi: 10.1016/j.procbio.2014.12.028, Process Biochemistry (en línea), 50(4), 589-597 (2015)

Valencia, P., Pinto, M. y Almonacid, S., Identification of the key mechanisms involved in the hydrolysis of fish protein by Alcalase, doi: 10.1016/j.procbio.2013.11.012, Process Biochemistry, 49(2), 258-264 (2014)

Wang, B., Li, L., Chi, C.F., Ma, J.H., Luo, H.Y. y Xu, Y.F., Purification and characterisation of a novel antioxidant peptide derived from blue mussel (Mytilus edulis) protein hydrolysate, doi:10.1016/j.foodchem.2012.12.002, Food Chemistry (en línea), 138(2-3), 1713-1719 (2013)

Yagoub, A., Mohamed, E., Ahmed, A. y El Tinay, A., Study on fururndu, a Traditional Sudanese fermented roselle (Hibiscus sabdariffa L.) seed: Effect on in vitro protein digestibility, chemical composition and functional properties of the total proteins, doi: 10.1021/jf0496548, Jounal Agricultural Food Chemistry (en línea), 52, 6143-6150 (2004)

Zhu, L., Chen, J., Tang, X. y Xiong, Y., Reducing, Radical Scavenging, and Chelation Properties of in Vitro Digests of Alcalase-Treated Zein Hydrolysate, doi: 10.1021/jf703697e, Journal Agricultural Food Chemistry (en línea), 56(8), 2714-2721 (2008) 
